\title{
Utilização de Ferramentas Colaborativas na Aprendizagem Musical de Alunos Leigos
}

\author{
Fernando Pinhati, Sean Siqueira
}

Departamento de Informática Aplicada - Universidade Federal do Estado do Rio de Janeiro Avenida Pasteur 458 - Urca, Rio de Janeiro - RJ, 22290-240

\{fernando.junior, sean\}@uniriotec.br

\begin{abstract}
This research aims at assessing the impact of the collaboration between inexperienced music students through educational activities involving music composition with computer support. A case study was conducted and it had both exploratory and explanatory parts. The exploratory aimed to compare the discourse between the two groups of students on the collaborative music composition activity. The explanatory part aimed to understand the participants' awareness about their individual production compared to their collaborative production. Results seem to indicate that the collaborative music composition activities are best seen by learners as compared to the same activities performed individually. Furthermore, we found that students seem to realize the "musical discourse" of his/her colleagues and reflect on it, adapting their individual contributions.
\end{abstract}

Resumo. A presente pesquisa tem como objetivo a avaliação das consequências da colaboração entre alunos leigos em música através de atividades educacionais envolvendo a composição musical com suporte computacional. Foi realizado um estudo de caso que teve uma parte exploratória e outra explanatória. A exploratória teve o objetivo de comparar o discurso entre os alunos de dois grupos na atividade de composição musical colaborativa realizada. Já a parte explanatória teve o objetivo de entender a percepção dos participantes sobre sua produção individual comparada a sua produção colaborativa. Um dos resultados parece indicar que atividades de aprendizagem de música por composição colaborativa são melhores vistas pelos aprendizes, quando comparadas às mesmas atividades executadas de maneira individual. Além disso, foi visto que os alunos parecem perceber o "discurso musical" de seus colegas e refletem sobre ele, adaptando suas contribuições individuais.

\section{Introdução}

Desde o final do século XX o modo de pensar, de se comunicar e, em geral, de viver das pessoas vem se modificando. A utilização de computadores pessoais e da internet têm grande responsabilidade sobre estas mudanças (WEI et al., 2011). Muitas atividades que antes eram realizadas sem o apoio direto de sistemas computacionais, hoje são realizadas com tal suporte e, muitas vezes, substituem completamente suas predecessoras não digitais. São exemplos clássicos: enviar e-mails, efetuar compras e conversar com amigos.

Estas modificações de agir e pensar também podem ser visualizadas na área da educação. Modelos de educação à distância com uso de sistemas computacionais 
começaram a aparecer e, por intermédio da internet, foram fortemente disseminados (RÓZEWSKI et al., 2011), fazendo inclusive com que o desenvolvimento de soluções de e-learning aparecesse como uma das áreas da educação que mais rápido se desenvolveram no final do século XX (GILBERT et al., 2007). Atualmente, o modelo é bastante utilizado com crianças e adultos, tanto na educação escolar quanto na corporativa.

O papel da colaboração na educação é conhecido. Existem diversas abordagens pedagógicas que consideram a colaboração como item fundamental no processo de construção do conhecimento. Teóricos da educação geral, como Vygotsky, e também da educação musical, como Swanwick, indicam que a colaboração entre os alunos deve ser estimulada no processo de aprendizagem (VYGOTSKY, 1962) (SWANWICK, 1979).

Um levantamento das pesquisas relacionadas ao ensino de música com suporte de sistemas computacionais publicadas nos congressos da ANPPOM (Associação Brasileira de Pesquisa em Pós-graduação de Música) e da ABEM (Associação Brasileira de Educação Musical) realizado por BRAGA \& RIBEIRO (2010) indica como uma das necessidades de pesquisa a investigação do impacto do uso de ferramentas computacionais colaborativas em atividades de educação musical.

Soma-se a este fato o crescimento de softwares colaborativos, específicos para o domínio musical, que podem ser utilizados no processo de aprendizagem do aluno de música (GOHN, 2010). Sistemas como NoteFlight ${ }^{1}, \mathrm{OhmStudio}^{2}$, Mantis ${ }^{3}$ e ambientes sociais como SoundCloud ${ }^{4}$, IndabaMusic ${ }^{5}$ e Musescore ${ }^{6}$ são exemplos de ferramentas com grande potencial colaborativo, criados recentemente e que mostram a ampliação da oferta destes tipos de software no mercado.

Segundo CROOK (1994), um dos benefícios que a tecnologia da informação traz na educação é o suporte a novos modos de aprendizagem, como por exemplo o colaborativo. $\mathrm{Na}$ área de tecnologias educacionais, a Aprendizagem Colaborativa com Suporte Computacional (CSCL) é um ramo que vem se destacando nas ciências da aprendizagem e que estuda a aprendizagem de grupos de pessoas por meio de sistemas que auxiliem na colaboração (STAHL et al., 2006). A CSCL requer a colaboração entre os alunos, fazendo com que eles não reajam isoladamente aos conteúdos repassados pelo professor, mas sim conjuntamente. A aprendizagem efetiva acontece através das interações entre os alunos, por meio de perguntas, compartilhamento de ideias, ensino mútuo e percepção de como os colegas aprendem. No caso de alunos leigos, uma das características importantes em um sistema educacional para este público é justamente a possibilidade de colaboração entre os aprendizes através das ferramentas disponibilizadas (PIMENTA et al., 2011).

Neste cenário, percebe-se que a colaboração é entendida como importante por teóricos e educadores musicais, porém existe uma lacuna a ser investigada quando esta colaboração é realizada através de sistemas computacionais. Ainda muito pouco é

\footnotetext{
${ }^{1}$ http://www.noteflight.com

${ }^{2} \mathrm{http}$ ://www.ohmstudio.com

${ }^{3} \mathrm{http}: / / \mathrm{www}$.indabamusic.com/help/mantis

${ }^{4} \mathrm{http}: / /$ www.soundcloud.com

${ }^{5} \mathrm{http}: / / \mathrm{www}$. indabamusic.com

${ }^{6} \mathrm{http}: / / \mathrm{www} . \mathrm{musescore.com}$
} 
conhecido sobre o processo colaborativo relacionado a atividades de composição mediada por computador, por exemplo (NIKOLAIDOU, 2011).

A presente pesquisa tem como objetivo a avaliação das consequências da colaboração, com uso de sistemas computacionais, entre alunos leigos através de atividades educacionais envolvendo a composição musical, que é uma das mais importantes atividades no processo de aprendizagem de música (SWANWICK, 1979). A ideia é fazer com que os participantes de um curso a distância realizem atividades de composição musical através de aplicativos de uso individual, onde só o próprio aluno estará criando sua música, e de aplicativos colaborativos, onde vários alunos estarão simultaneamente compondo uma mesma música. Os itens que se deseja observar são as consequências da colaboração na composição no que diz respeito à reflexão individual e à negociação entre os alunos e qual é a percepção deles sobre o assunto.

O texto está organizado em mais quatro seções. Na seção 2 será apresentado o modelo de análise de discurso utilizado no trabalho. O estudo de caso realizado para esta pesquisa é descrito na seção 3. A seção 4 discute sobre os resultados obtidos. Por fim, na seção 5, são apresentadas as considerações finais do trabalho.

\section{Análise de Discurso em Atividades Musicais Colaborativas}

O conhecimento é construído em um contexto de trocas, onde se tem o conhecimento atual, já sabido pela pessoa, e as dúvidas que aparecem sobre estes conhecimentos, ocasionadas pelas mudanças no mundo e vivências de situações ainda não entendidas. Este desequilíbrio entre conhecido e conhecido, entre certezas e dúvidas, faz com que sejam estabelecidas novas relações ou conhecimentos (FAGUNDES et al., 1999).

$\mathrm{Na}$ aprendizagem colaborativa, o que se procura é o trabalho conjunto entre os estudantes, tornando-os responsáveis pela sua própria aprendizagem e também pela dos demais alunos. Nesta situação, o conhecimento se constrói através de explicações, debates, argumentações e das trocas de experiências entre todas as pessoas do grupo (CASTRO \& MENEZES, 2011).

Alguns pesquisadores trazem justificativas para o uso de sistemas colaborativos na educação musical, no que tange a atividade de composição. A troca de conhecimento derivada das possibilidades de improvisação de cada aluno é importante no processo de aprendizagem (BURNARD \& YOUNKER, 2008). Além disso, através da interação entre os alunos eles se tornam mais socializados segundo a teoria de desenvolvimento sócio-cultural de VYGOTSKY (1962), consequência benéfica principalmente quando se trata de crianças como aprendizes.

É importante o diálogo na colaboração. É a discussão que proporciona a troca de idéias e promove a consolidação de pontos de vistas alternativos. Segundo STAHL et al. (2006), uma das formas de avaliar as consequências de atividades colaborativas é através da análise do discurso decorrente da execução de um estudo de caso, do qual tenham sido coletados dados como textos, áudios ou vídeos que relatem a comunicação entre os participantes. Para análise do discurso aplicado ao domínio da educação musical, NIKOLAIDOU (2011) propõe o modelo ComPLuS.

O modelo ComPLuS define um modelo de análise de discurso em atividades de composição colaborativa e associa cada tipo fala a várias possibilidades de ações a 
serem tomadas. Na presente pesquisa, apenas o modelo de análise de discurso será utilizado para realização da classificação das mensagens trocadas pelos alunos nas atividades de composição colaborativa.

São cinco tipos de diálogos enumerados por NIKOLAIDOU (2011): Disputacional (DISP), Acumulativa (CUM), Exploratória (EXP), Reflexiva (REF) e Operacional (OPER).

Os diálogos do tipo disputacionais são identificados em contextos onde não existe evidência de consenso entre os participantes. São caracterizados por desacordos (DIS) e propostas seguidas de ações individuais (PAI), por exemplo.

Os acumulativos indicam que existe algum tipo de esforço para atingir um consenso. É um tipo de discurso de negociação. São caracterizados por ofertas de novas propostas (PO), perguntas sobre novas propostas (PQ), comentários (COM), acordos (AGR), confirmações $(\mathrm{CON})$ e contrapropostas $(\mathrm{CP})$, por exemplo.

O tipo exploratório de discurso é caracterizado pela evidência de debate entre os participantes e da exploração conjunta na busca por melhores soluções. Críticas (CRI), clarificações (CLAR), explicações (EXPL), perguntas buscando por clarificações (QCLAR) e propostas seguidas por uma ação acordada (PAC) são exemplos deste tipo de fala. Também faz parte do discurso de negociação.

Os diálogos do tipo reflexivo indicam que as pessoas estão tentando expressar criticamente e construtivamente uma reflexão sobre um pensamento próprio. São caracterizados por perguntas reflexivas (SQ) e julgamento de próprias ações (SE).

O tipo operacional de discurso é caracterizado pelas conversações a respeito de questões relacionadas ou a tarefa (UT) em si ou ao software (US).

\section{Estudo de Caso}

Para análise da hipótese deste trabalho, um estudo de caso foi realizado com cinco alunos, todos leigos em música. Estes alunos foram divididos em dois grupos, um com três pessoas - Grupo I - e outro com duas pessoas - Grupo II. Os participantes escolhidos tinham idades distintas, sendo três entre 20 e 30, um entre 30 a 40 e mais um entre 50 a 60 anos. Em relação ao gênero, participaram três homens e duas mulheres. Os grupos foram divididos de forma a misturar as idades e os gêneros.

Um curso sobre parâmetros básicos do som foi criado em uma plataforma de ensino a distância. O curso consistia no ensino de conceitos básicos do som, como altura, intensidade, timbre, duração e ritmo. Este conteúdo foi dividido em dois módulos:

- Módulo I - Altura e Intensidade.

- Módulo II - Ritmo, Duração e Timbre.

É importante ressaltar que os conceitos apresentados em cada módulo são independentes entre si, de forma que a ordem de execução dos módulos não é importante. 
O material didático do curso foi constituído de objetos de aprendizagem coletados do Portal do Professor ${ }^{7}$ e a partir de uma página de jogos para aprendizagem musical ${ }^{8}$. Um resumo de cada conceito também foi disponibilizado para cada módulo. O objetivo do material era que o aluno absorvesse os conceitos repassados de forma prática, para que depois pudesse utilizar tal conhecimento em uma composição musical.

Como atividade de fim de módulo, cada aluno deveria compor uma música utilizando os conceitos aprendidos. Como software para composição, era indicado o EditorMusical (FICHEMAN et al., 2003), que possui tanto uma versão individual quanto uma colaborativa para criação musical.

O fato de possuir um ambiente não formal de composição, ou seja, um ambiente onde não é necessário saber conceitos de notação musical formal para criar melodias, assim como o fato de possuir duas versões, uma individual e outra colaborativa, com interface e recursos semelhantes foram determinantes para a escolha do EditorMusical para o estudo de caso desta pesquisa.

A execução dos módulos pelos grupos foi alternada. Enquanto o Grupo I fez o Módulo I primeiro, o Grupo II fez o Módulo II. Nesta primeira parte, cada aluno de cada grupo teve como atividade final a criação de uma música utilizando o EditorMusical em seu modo individual. Já na segunda etapa, cada grupo teve como atividade uma criação musical utilizando também o EditorMusical, mas em seu modo colaborativo. O desenho da pesquisa é apresentado na figura 1.

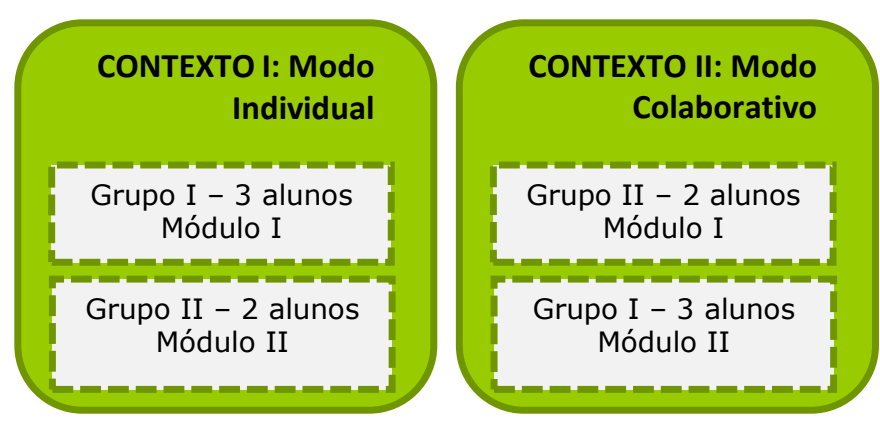

Figura 1 - Desenho da Pesquisa

A unidade de análise foi a execução de cada módulo por ambos os grupos. $\mathrm{O}$ estudo de caso teve uma parte exploratória e outra explanatória. O estudo exploratório teve o objetivo de comparar o discurso entre os alunos dos dois grupos na atividade de composição musical colaborativa realizada. Os dados para análise do discurso foram coletados manualmente do log do bate-papo (chat) existente no modo de composição colaborativa do EditorMusical e analisados através do framework ComPlus de análise do discurso musical (NIKOLAIDOU, 2011).

Já a parte explanatória teve o objetivo de entender a percepção dos participantes sobre sua produção individual comparada a sua produção colaborativa. Para isso, foi elaborado um questionário com quatro perguntas objetivas, com opções de resposta em escala Likert, e quatro perguntas abertas, estas utilizadas para entender melhor as respostas objetivas dos participantes. Foi realizada uma análise quantitativa em cima das

\footnotetext{
${ }^{7} \mathrm{http}: / /$ portaldoprofessor.mec.gov.br/index.html

${ }^{8} \mathrm{http}: / /$ jogosmusicais.blogspot.com/
} 
respostas às questões objetivas do questionário. Os resultados estão detalhados na próxima seção.

\section{Resultados}

Quanto à avaliação dos alunos sobre a percepção de qualidade em suas próprias composições, é possível verificar que existe uma tendência de preferência pelas criações realizadas no modo colaborativo (figura 2). Apenas composições neste modo foram percebidas como excelentes pelos alunos e as criações realizadas no modo individual foram avaliadas mais negativamente.

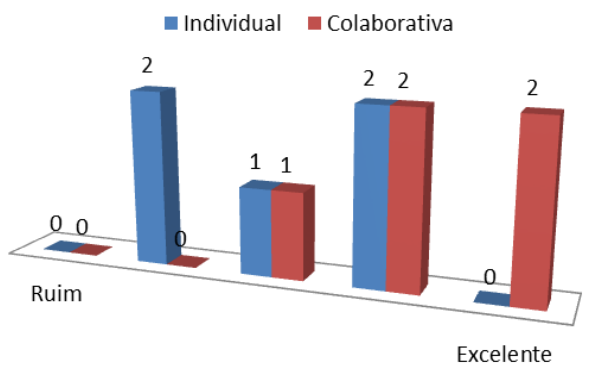

Figura 1 - Auto-avaliação da qualidade da composição

Em relação ao número de modificações realizadas na composição até que fosse obtido um resultado satisfatório - o que inclui ações de experimentação, tentativa e erro, reflexão sobre o que está sendo produzido e negociação com os demais alunos (neste caso no modo colaborativo) - percebe-se que os dois modos possuem uma distribuição semelhante na visão dos alunos (figura 3). Porém, o fato a ressaltar é que no modo colaborativo existe uma tendência de aumento do número de modificações, enquanto no modo individual nenhum aluno indica que modificou sua criação muitas vezes.

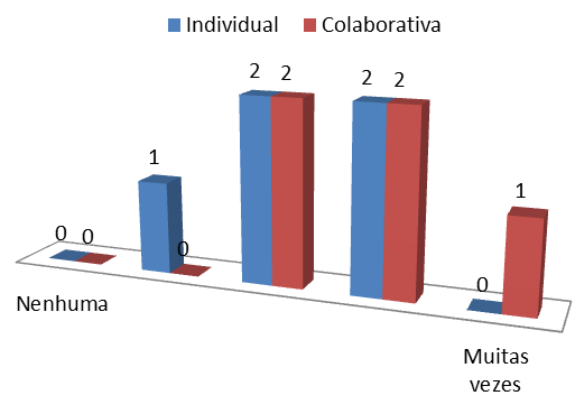

Figura 2 - Número de modificações durante a composição

Sobre a preferência dos alunos em relação ao modo de composição, individual ou colaborativo, a maioria indica gostar da mesma forma dos dois modelos (figura 4). 

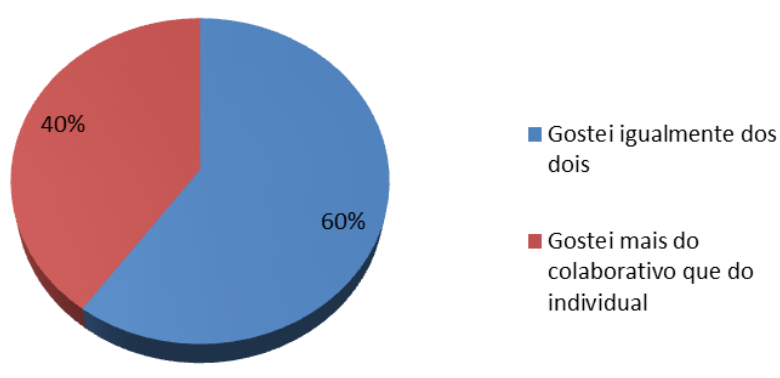

Figura 3 - Preferência entre modo individual e colaborativo

Mesmo sem uma preferência maior do editor no modo colaborativo, é possível perceber que os alunos avaliam melhor as composições realizadas nele e que as atividades que geram modificações na criação, como debate e reflexão, parecem ser maiores neste modo, já que o número de modificações é maior.

Ao considerar a justificativa de preferência dos alunos através das respostas às perguntas abertas, é possível verificar que três participantes parecem indicar que o modo colaborativo possibilita uma maior reflexão sobre suas próprias ações. Tal fato pode ser constatado quando os alunos respondem que ao ouvirem sons construídos por outras pessoas do grupo tentam acompanhá-los, refinando sua contribuição ou ainda aprendendo como o colega está utilizando a ferramenta para alcançar seus objetivos. Outras duas pessoas indicam que o modo colaborativo é mais divertido, mesmo tendo indicado a preferência igual entre os modos.

O discurso no modo colaborativo foi analisado através da aplicação do framework ComPlus (NIKOLAIDOU, 2011). As figuras 5 e 6 ilustram a distribuição dos tipos de discursos da dinâmica colaborativa realizada com cada grupo.

As falas relacionadas ao operacional são relativas às dúvidas de manipulação de funcionalidades do software e de percepção de contribuições dos colegas como, por exemplo, qual cor que representava cada instrumento, quem estava compondo o que em determinado momento, dúvidas de execução de algumas funcionalidades, etc. Nos dois grupos, este tipo de fala apareceu com bastante frequência, indicando dúvidas na manipulação e no entendimento das funcionalidades do software utilizado.

Os discursos relacionados à reflexão e a exploração de ideias aparecem com igual percentual tanto na dinâmica com o Grupo I quanto na atividade com o Grupo II. Este fato é interessante, pois mesmo com a diferença de número de participantes e de perfil de pessoas, as atividades de reflexão e exploração pareceram possuir igual destaque no discurso dos alunos. 


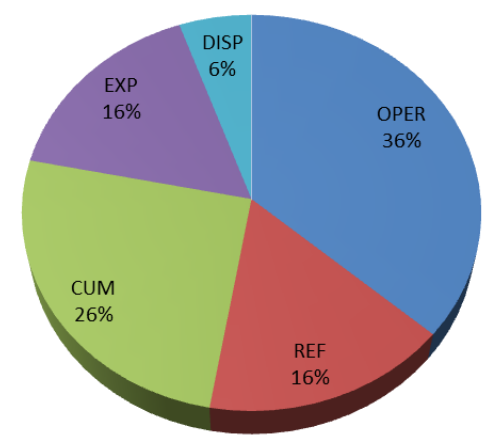

Figura 4 - Classificação do discurso colaborativo - Grupo I

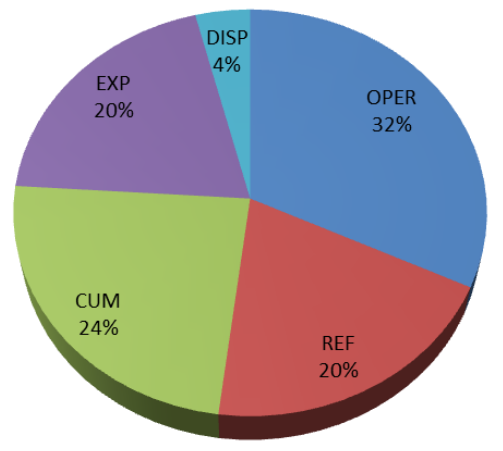

Figura 5 - Classificação do discurso colaborativo - Grupo II

As falas de exploração (EXP), caracterizadas pelo debate entre os pares e na exploração conjunta para melhoria da composição, e as de acumulação (CUM), caracterizadas pelo esforço para alcance de consenso, são discursos típicos de negociação. Somando seus percentuais é possível perceber que nos dois grupos os discursos de negociação superam os de reflexão (REF), onde o aluno age de forma crítica e construtiva expressando seus pensamentos. Isto parece indicar que os alunos estavam mais determinados com a realização da tarefa de forma cooperativa, preocupando-se também com a preferência de seus colegas e debatendo sobre a qualidade da composição resultante, do que com a exposição de suas ideias ou o compartilhamento de suas reflexões sobre a composição com os demais participantes.

Nos dois grupos, os discursos relacionados à disputa (DISP), onde existe uma competição a respeito de qual ideia que será utilizada, apareceram com frequência menor.

Outro tipo de comunicação que aparece e é deixado claro pelos alunos dos dois grupos é a comunicação musical. Os alunos dos dois grupos parecem perceber que a parte composta pelos colegas faz parte de sua comunicação e tentam se adequar a ela através da sua contribuição pessoal. Tanto no discurso da dinâmica colaborativa quanto nas respostas às perguntas abertas do questionário, é possível verificar que os alunos parecem se entrosar um com o outro musicalmente, depois de decorrido certo tempo de composição conjunta. A análise deste fator também parece indicar um aumento na reflexão e na negociação do aluno com seus colegas, porém na forma de comunicação musical e não textual. 
É importante ressaltar que houve estímulo do tutor para que os alunos conversassem mais no chat. Alguns julgamentos dos alunos sobre sua própria produção (SE - Self Elaboration, no modelo ComPlus) foram resultados destes estímulos.

Outra importante ressalva é que os alunos estavam localizados em regiões remotamente diferentes, comunicando-se apenas através do chat disponibilizado pelo software que, como os próprios participantes ressaltaram, é muito pequeno e difícil de acompanhar.

Apesar dos alunos já terem utilizado a mesma ferramenta na dinâmica individual, fato que poderia influenciar no domínio da ferramenta por parte do aluno, a maioria dos alunos se mostraram ainda em um nível experimental da ferramenta mesmo na dinâmica colaborativa. Apenas um aluno ressaltou que já tinha um conhecimento melhor da ferramenta adquirido através da tarefa individual.

\section{Considerações Finais}

Como resultado da análise das respostas aos questionários, é possível verificar que os alunos avaliam de forma melhor suas composições criadas de forma colaborativa do que as criadas individualmente, o que parece indicar que atividades de aprendizagem de música por composição colaborativa são melhores vistas pelos aprendizes, quando comparadas às mesmas atividades, porém, individuais.

De forma exploratória, foi percebido que a distribuição das categorias de diálogos nos discursos de grupos com perfis totalmente diferentes são bem semelhantes. Além disso, a análise aponta que os discursos de negociação superaram os de reflexão na atividade colaborativa, o que parece indicar que a composição final foi afetada pela colaboração e de forma positiva, quando é juntado este resultado com a percepção da qualidade da composição pelos alunos.

Outro resultado interessante desta pesquisa é a questão da comunicação através do meio musical. Foi visto que os alunos parecem perceber o "discurso musical" de seus colegas e refletem sobre ele, adaptando suas contribuições individuais. Nas dinâmicas realizadas, os alunos puderam trocar ideias sobre todos os conceitos através da dinâmica colaborativa de composição, tanto de forma textual quanto de forma musical.

A comunicação musical identificada na pesquisa vai além da análise do discurso textual tradicional e pode ser objeto de pesquisas futuras. Trabalhos que procurem saber qual é a influência da comunicação musical no aprendizado e na criação do aluno são indicados aqui como possíveis continuações desta pesquisa.

Outro trabalho futuro que pode ser realizado é a avaliação das composições musicais produzidas tanto no modo individual quanto no colaborativo segundo uma teoria de desenvolvimento musical, como a Teoria do Espiral de SWANWICK et al. (1986). Tal avaliação indicaria se o uso de ferramentas colaborativas pode influenciar também no desenvolvimento musical do aluno.

\section{Referências}

Braga, P. D. A., \& Ribeiro, G. M. (2010). Lições de Interação em um Curso de Violão a Distância. XIX Congresso Nacional da ABEM (pp. 1168-1176). Goiânia. 
Burnard, P., Younker, B. A. (2008). Investigating children's musical interactions within the activities systems of group composing and arranging: an application of Engestrom's activity theory. International Journalof Educational Research, 47(1), 6074.

Castro, A., Menezes, C. (2011). Aprendizagem Colaborativa com Suporte Computacional. In: Sistemas Colaborativos. Elsevier.

Crook, C. (1994). Computers and the collaborative experience of learning. London: Routledge.

Fagundes, L. C., Sato, L. S., Maçada, D. L. (1999). Aprendizes do Futuro - as inovações já começaram. Brasília, MEC.

Ficheman, I. K., Lipas, R. A., Krüger, S. E., Lopes, R. D. D. (2003).Editor Musical : uma Aplicação para a Aprendizagem de Música apoiada por Meios Eletrônicos Interativos. Simpósio Brasileiro de Informática na Educação (pp. 176-185). Rio de Janeiro.

Gilbert, J., Morton, S., \& Rowley, J. (2007). e-Learning: The student experience. British Journal of Educational Technology, 38(4), 560-573. doi:10.1111/j.14678535.2007.00723.x

Gohn, D. (2010). Tendências na educação à distância: os softwares on-line de música. Opus, 16, 113-126.

Nikolaidou, G. N. (2011). ComPLuS Model: A new insight in pupils' collaborative talk, actions and balance during a computer-mediated music task. Computers \& Education. Elsevier Ltd.

Pimenta, M., Miletto, E., Flores, L., \& Hoppe, A. (2011). Cooperative mechanisms for networked music. Future Generation Computer Systems, 27(1), 100-108. Elsevier B.V. doi: 10.1016/j.future.2010.03.005.

Rózewski, P., Kusztina, E., Tadeusiewicz, R., \& Zaikin, O. (2011). Intelligent Open Learning Systems: Concepts, Models and Algorithms. Springer.

Stahl, G., Koshcmann, T., Suthers, D. (2006). Computer-Supported Collaborative Learning. In: R. Keith Sawyer (org.) The Cambridge Handbook of the Learning Sciences. Cambridge Un. Press.

Swanwick, K. (1979) A Basis for Music Education. London: Routledge.

Swanwick, K., Tillman, J. (1986) The sequence of musical development: a study of children's composition, British Journal of Music Education, 3, p.305-339. Cambridge: Cambridge University Press.

Vygotsky, L. S. (1962). Thought and Language (E. Hanfmann \& G. Vakar, Eds. \& Trans.). Cambridge, MA: MIT Press.

Wei, C.-T., \& Young, S. S. C. (2011). Investigating the Role and Potentials of Using Web2.0 in Music Education from Student Perspective. 2011 IEEE 11th International Conference on Advanced Learning Technologies, 344-346. doi:10.1109/ICALT.2011.107 